\title{
Direct magnetic resonance arthrography of the canine elbow
}

\author{
Yauheni Zhalniarovich, Paulina Przyborowska-Zhalniarovich, \\ Marta Mieszkowska, Zbigniew Adamiak \\ University of Warmia and Mazury in Olsztyn, Faculty of Veterinary Medicine, \\ Department of Surgery and Radiology, Olsztyn, Poland
}

Received July 26, 2016

Accepted February 17, 2017

\begin{abstract}
This study compares the effects of four dilutions of the gadolinium-containing contrast media $(1: 100 ; 1: 400 ; 1: 800 ; 1: 1,200)$ administered to the elbow on the quality of magnetic resonance images. All the examined dilutions had a positive effect on image quality, and 1:800 was regarded as the optimal dilution of gadolinium for viewing the elbow because it imparted good contrast to the joint cavity without obliterating the contours of articular surfaces. Transverse, sagittal, and dorsal low-field magnetic resonance images were obtained in 24 canine cadaver front limbs. The musculus biceps brachii, $\mathrm{m}$. triceps brachii, m. extensor carpi radialis, m. flexor carpi ulnaris, the articular surfaces, the medial coronoid process and the anconeal process of the ulna were well visualized by High Resolution Gradient Echo, XBONE T2 and Spin Echo T1 sequences in the sagittal plane. The biceps brachii, pronator teres, flexor carpi radialis, extensor digitorum communis, extensor carpi radialis, deltoid muscle and the articular surface of the medial condyle of the humerus were very well visualized by 3D SST1 and XBONE T2 sequences in the transverse plane. The triceps brachii muscle, extensor digitorum lateralis muscle, superficial digital flexor, deep digital flexor and the medial condyle of the humerus were very well visualized by the Spin Echo T1 sequence in the dorsal plane. This article describes for the first time the use of the gadolinium contrast agent administered to the canine elbow joint during magnetic resonance modality. Magnetic resonance arthrography can be a helpful visualization technique in treating canine soft tissue elbow injury.
\end{abstract}

MRI, contrast agent, gadolinium, dogs

Magnetic resonance imaging (MRI) is a method of choice for diagnosing elbow joint disorders in humans. It is one of the recently developed diagnostic techniques (Dalinka et al. 1989; De Smet et al. 1990; Hayes and Conway 1992; Hodler et al. 1992; Heron and Calvert 1992; Recht 1994; Buckwalter 1996; Hill et al. 2000; Sahin and Demirtas 2006).

In human medicine, MRI is used to diagnose free-floating fragments in joints, and it is highly useful in evaluations of collateral ligaments (Hill et al. 2000). The contrast agents can be administered directly to the joint cavity, which is an additional advantage of MRI (Hill et al. 2000). Diluted gadolinium-containing compounds are the most popular contrast agents for MRI (Tirman et al. 1993; Winalski et al. 1993).

Magnetic resonance imaging supports evaluations of the thickness and structure of articular cartilage. Minor damage to articular cartilage, the joint capsule and the surrounding soft tissues cannot be effectively evaluated by standard MRI. Contrast agents are administered by intra-articular injection to enhance the visualization of the joint cavity (Recht et al. 2005; Sahin et al. 2006; Gold et al. 2009). Magnetic resonance contrast agents all contain the paramagnetic component gadolinium, and their mechanisms are based on the same principle of physics (Kuriashkin and Losonsky 2000).

The first objective of this study was to compare the effect of four dilution levels of the gadolinium contrast agent (Omniscan) administered to the elbow joint cavity on the quality

Address for correspondence:

Yauheni Zhalniarovich

Department of Surgery and Radiology

University of Warmia and Mazury in Olsztyn

Oczapowskiego 14, 10-957 Olsztyn, Poland

Phone: +48 895233795

E-mail: eugeniusz.zolnierowicz@uwm.edu.pl

http://actavet.vfu.cz/ 
of MR images. The second objective was to determine the optimal level of gadolinium contrast medium dilution in MRI examinations of the canine elbow joint.

\section{Materials and Methods}

The study was performed on 24 elbow joints of 12 dogs of both sexes euthanized for reasons unrelated to orthopedic conditions or this study (4 German shepherds, 3 Labradors, 1 Doberman and 4 crossbreeds). All were aged 2 to 4 years, with body weights of 25 to $37 \mathrm{~kg}$ (mean weight $31 \mathrm{~kg}$ ). None of the patients had a history of elbow joint disease. None of the dogs showed lameness of the front limbs for 8 weeks before euthanasia. During orthopedic examination, the limbs showed no pain and were within a normal range of motion. No evident abnormalities were observed over standard radiographic examinations. Both elbows of each dog were subjected to MRI immediately after euthanasia.

The patients were examined in a low-field $(0.25 \mathrm{~T})$ MRI scanner with a permanent magnet (Vet-MR Grande, Esaote, Italy), using the ankle/foot DPA coil used in human medicine. All patients were positioned in lateral recumbency with the elbow joint extended and centered in the middle of the coil. The elbow joint was scanned in three planes: sagittal, transverse and dorsal. Three sequences were selected in the sagittal plane: High Resolution Gradient Echo (TR 700-800 ms/ TE $18 \mathrm{~ms}$ ), XBONE T2 (TR 900-1000/ TE 17-19 ms) and Spin Echo T1 (TR 700-900 ms/TE 18-20 ms). The Spin Echo T1 sequence was used in the dorsal plane. Two sequences were applied in the transverse plane: 3D SST1 and XBONE T2. In all sequences, slice thickness was $2 \mathrm{~mm}$ with 0 mm distance between slices. Scanning time was 35-40 min.

After standard MRI modality the examination was repeated with the use of the gadolinium contrast agent (Omniscan, GE Healthcare, USA, $0.5 \mathrm{mmol} / \mathrm{ml}$ ) administered to the joint cavity at four dilution levels (1:100; $1: 400 ; 1: 800 ; 1: 1,200)$ in $0.9 \% \mathrm{NaCl}$ to determine the effect of the contrast agent on the quality of MR images. In conversion the molar concentrations were $5,1.25,0.625,0.42$, respectively. The contrast solution was administered at the amount of $1-2 \mathrm{ml}$, subject to the size of the visualized joint. After the administration of the contrast agent, the joint was extended and flexed for $60 \mathrm{~s}$ to distribute the solution evenly in the joint cavity. After $60 \mathrm{~s}$, the elbow joint was placed inside the MRI coil, positioned, and scanned. For this contrast examination, the patients were divided into four groups of six elbows each. In subgroup 1, the contrast agent was diluted 1:100; in subgroup $2-1: 400$; in subgroup $3-1: 800$; and in subgroup $4-1: 1,200$. Images were evaluated for intensity of the contrast agent relative to the surrounding tissues, joint space brightness, and homogeneous consistency of the contrast medium. All obtained images were rated by all co-authors of the publication.

\section{Results}

The contrast agent administered to the elbow joint cavity improved the quality of the resulting images and enhanced the visualization of articular surfaces. All analyzed dilutions of the Omniscan contrast agent $(1: 100 ; 1: 400 ; 1: 800 ; 1: 1,200)$ supported a more detailed examination of the elbow joint cavity. Omniscan dilution of 1:800 produced MR images of the most satisfactory quality. High Resolution Gradient Echo, XBONE T2 and Spin Echo T1 sequences in the sagittal plane proved to be highly useful for evaluating structures such as the medial coronoid process and anconeal process of the ulna and articular surfaces of the elbow joint. The contrast agent is injected to expand the joint space and produce hyperintense images. It enhances the visualization of articular surfaces, the joint capsule, and the surrounding soft tissues. Sequences in the sagittal plane are applied in examinations of the biceps brachii muscle, triceps brachii muscle, extensor carpi radialis muscle and flexor carpi radialis muscle (Plate IV, Fig. 1).

The administration of a contrast agent in the transverse plane (3D SST1 and XBONE T2) and the dorsal plane (Spin Echo T1) enhances the visualization of humeral condyles and their articular surfaces. The contrast agent fills the joint cavity and brings out the contours of articular surfaces. The biceps brachii muscle, pronator teres muscle, flexor carpi radialis muscle, extensor digitorum communis muscle, extensor carpi radialis muscle and deltoid muscle were also very well visualized by sequences in the transverse plane (Plate V, Fig. 2). The triceps brachii muscle, extensor digitorum lateralis muscle, superficial digital flexor and deep digital flexor were very well visualized by the Spin Echo T1 sequence in the dorsal plane (Plate V, Fig. 3).

The quality of MR images was enhanced even when the contrast agent was administered at the most diluted solution of 1:1,200. The contrast medium leads to the mechanical 
widening of the elbow joint which is very tight in dogs. Gadolinium also provides additional contrast by adhering to articular surfaces and filling the joint cavity.

\section{Discussion}

Magnetic resonance imaging is a modern, non-invasive and highly sensitive diagnostic tool for evaluating articular surfaces and joint cavities (Konig et al. 1987; Solomon et al. 1989; Tervoven et al. 1993; Snaps et al. 1997; Miller 1999; Recht et al. 2005; Janach et al. 2006; Schaefer and Forrest 2006; Probst et al. 2007; Agnello et al. 2008; Gold et al. 2009; Jaskolska et al. 2013).

In our study, all analyzed dilutions of the contrast agent improved the quality of MR images. The contrast medium should be evenly distributed in the entire joint cavity. In the present experiment, this was achieved by flexing and extending the elbow joint for around $60 \mathrm{~s}$. Image quality is determined by the time of joint manipulation after the administration of the contrast agent. Longer manipulation time can improve image quality (Reichle and Snaps 1999; Probst et al. 2007).

The biceps brachii muscle, triceps brachii muscle, extensor carpi radialis muscle and flexor carpi ulnaris muscle were very well visualized by High Resolution Gradient Echo, XBONE T2 and Spin Echo T1 sequences in the sagittal plane. Those sequences were also highly useful in evaluations of articular surfaces of the elbow joint, the medial coronoid process and the anconeal process of the ulna. The biceps brachii muscle, pronator teres muscle, flexor carpi radialis muscle, extensor digitorum communis muscle, extensor carpi radialis muscle and deltoid muscle were very well visualized by 3D SST1 and XBONE T2 sequences in the transverse plane. Those sequences also supported detailed visualization of articular surfaces of the medial condyle of the humerus. The Spin Echo T1 sequence in the dorsal plane was highly effective in diagnosing the triceps brachii muscle, extensor digitorum lateralis muscle, superficial digital flexor, deep digital flexor and the medial condyle of the humerus.

It was previously recommended to use the gadolinium solutions in MRI examinations (Tirman et al. 1993; Winalski et al. 1993). According to Van Bree et al. (1995) and Murphy et al. (2008), the administration of contrast to the joint cavity enhances the visibility of soft tissues and articular surfaces. In some works, the administration of diluted gadolinium supported detailed imaging of articular surfaces (Gray et al. 2008; Guermazi et al. 2008; Taylor et al. 2009). Schaefer et al. (2010) performed MRI scans of the canine shoulder joint by administering the diluted gadolinium contrast agent $(1: 100 ; 1: 400 ; 1: 800$; $1: 1,000 ; 1: 1,200 ; 1: 1,400 ; 1: 1,600)$ directly to the joint. In the above study, $1: 1,200$ was the optimal dilution level which imparted very good contrast to the joint cavity and ligaments without obliterating the contours of articular surfaces (Schaefer et al. 2010). In our experiment, Omniscan (gadolinium) contrast agent delivered superior contrast resolution in examinations of the elbow joint. In this study, 1:800 was regarded as the optimal dilution level for a detailed evaluation of elbow joint anatomy.

Local safety proved to be superior after joint administration of gadolinium and rapid renal excretion took place (Schulte-Altedorneburg et al. 2003). An analysis of clinical studies spanning over 14 years (1987-2001), where gadolinium was administered to human subjects by intra-articular injection, revealed that Omniscan did not deliver toxic effects (Schulte-Altedorneburg et al. 2003).

Our results confirm the findings of other authors (Agnello et al. 2008; Murphy et al. 2008; Schaefer et al. 2010) who demonstrated that MRI is a highly useful tool for evaluating soft tissues and articular surfaces in examinations of the canine elbow joint. The administration of the gadolinium contrast agent significantly enhances the quality of MR images. In this study, 1:800 was regarded as the optimal dilution level for a detailed 
assessment of elbow joint anatomy. Our outcomes agree with previous studies (Agnello et al. 2008; Murphy et al. 2008; Schaefer et al. 2010) that direct MR arthrography might be useful for the assessment of canine soft tissue elbow lesions.

\section{References}

Agnello KA, Puchalski SM, Wisner ER, Schulz KS, Kapatkin AS 2008: Effect of positioning, scan plane and arthrography on visibility of periarticular canine shoulder soft tissue structures on magnetic resonance images. Vet Radiol Ultrasound 49: 529-539

Buckwalter KA 1996: Magnetic resonance of the knee. Radiologist 3: 99-122

Dalinka MK, Kricun ME, Zlatkin MB, Hibbard CA 1989: Modern diagnostic imaging in joint disease. AJR Am J Roentgenol 152: 229-240

De Smet AA, Fisher DR, Graf BK, Lange RH 1990: Osteochondritis dissecans of the knee: value of MR imaging in determining lesion stability and the presence of cartilage defects. Am J Roentgen 155: 549-553

Gold GE, Chen CA, Koo S, Hargreaves BA, Bangerter NK 2009: Recent advances in MRI of articular cartilage. Am J Roentgenol 193: 628-638

Gray ML, Burstein D, Kim YJ, Maroudas A 2008: Magnetic resonance imaging of cartilage glycosaminoglycan: basic principles, imaging technique, and clinical applications. J Orthop Res 26: 281-291

Guermazi A, Burstein D, Conaghan P, Eckstein F, Hellio Le Graverand-Gastineau MP, Keen H, Roemer FW 2008: Imaging in osteoarthritis. Rheum Dis Clin North Am 34: 645-687

Hayes CW, Conway WF 1992: Evaluation of articular-cartilage - radiographic and cross-sectional imaging techniques. Radiographics 12: 409-428

Heron CW, Calvert PT 1992: Three-dimensional gradient echo MR imaging of the knee: Comparison with arthroscopy in 100 patients. Radiology 183: 839-844

Hill NB, Bucchieri JS, Shon F, Miller TT, Rosenwasser MP 2000: Magnetic resonance imaging of the injury to the medial collateral ligament of the elbow: a cadaver model. J Shoulder Elbow Surg 9: 418-422

Hodler J, Berthiaume MJ, Scheitzer ME, Resnick D 1992: Knee joint hyaline cartilage defects: A comparative study of MR imaging and anatomic sections. J Comput Assist Tomo 16: 597-603

Janach KJ, Breit SM, Kunzel WW 2006: Assessment of the geometry of the cubital (elbow) joint of dogs by use of magnetic resonance imaging. Am J Vet Res 67: 211-218

Jaskolska M, Adamiak Z, Zhalniarovich Y, Holak P, Przyborowska P 2013: Magnetic resonance protocols in equine lameness examination, used sequences, and interpretation. Pol J Vet Sci 16: 803-811

Konig H, Sauter R, Deimling M, Vogt M 1987: Cartilage disorders: comparison of spin-echo CHESS, and FLASH sequence MR images. Radiology 164: 753-758

Kuriashkin IV, Losonsky JM 2006: Contrast enhancement in magnetic resonance imaging using intravenous paramagnetic contrast media: a review. Vet Radiol Ultrasound. 41: 4-7

Miller TT 1999: Imaging of elbow disorders. Orthop Clin North Am 30: 21-36

Murphy SE, Ballegeer EZ, Forrest LR, Schaefer SL 2008: Magnetic resonance imaging findings in dogs with confirmed shoulder pathology. Vet Surg 37: 631-638

Probst A, Modler F, Kunzel W, Mlynarik V, Trattnig S 2007: Demonstration of the articular cartilage of the canine ulnar trochlear notch using high-field magnetic resonance imaging. Vet J 177: 63-70

Recht MP 1994: MR imaging of articular cartilage: current status and future directions. AJR Am J Roentgenol 24: $283-290$

Recht MP, Goodwin DW, Winalski CS, White LM 2005: MRI of articular cartilage: Revisiting current status and future directions. Am J Roentgenol 185: 899-914

Reichle JK, Snaps F 1999: The elbow. Clin Tech Small Anim Prac 14: 177-186

Sahin G, Demirtas M 2006: An overview of MR arthrography with emphasis on the current technique and applicational hints and tips. Eur J Radiol 58: 416-430

Schaefer SL, Forrest LJ 2006: Magnetic resonance imaging of the canine shoulder: an anatomic study. Vet Surg 35: 721-728

Schaefer SL, Baumel CA, Gerbig JR, Forrest LJ 2010: Direct magnetic resonance artrography of the canine shoulder. Vet Radiol Ultrasound 51: 391-396

Schulte-Altedorneburg G, Gebhard M, Wohlgemuth WA, Fischer W, Zentner J, Wegener R, Balzer T, Bohndorf K 2003: MR arthrography: pharmacology efficacy and safety clinical trials. Skeletal Radiol 32: 1-12

Snaps FR, Balligand MH, Saunders JH, Park RD, Dondelinger RF 1997: Comparison of radiography, magnetic resonance imaging, and surgical findings in dogs with elbow dysplasia. Am J Vet Res 58: 1367-1370

Solomon SL, Totty WG, Lee JK 1989: MR imaging of the knee: comparison of the three-dimensional FISP and two-dimensional spin-echo pulse sequences. Radiology 173: 739-742

Taylor C, Carballido-Gamio J, Majumdar S, Li X 2009: Comparison of quantitative imaging of cartilage for osteoarthritis: T2, T1 rho, dGEMRIC and contrast-enhanced computer tomography. Magn Reson Imaging 27: 779-784

Tervoven O, Dietz MJ, Carmichael SW, Ehman RL 1993: MR imaging of knee hyaline cartilage: evaluation of two-and three-dimensional sequences. J Magn Reson Imaging 3: 663-668 
Tirman PF, Stauffer AE, Crues JV, Turner RM, Nottage WM, Schobert WE, Rubin BD, Janzen DL, Linares RC 1993: Saline magnetic resonance arthrography in the evaluation of glenohumeral instability. Arthroscopy 9: 550-559

Van Bree H, Van Ryssen B, Degryse H, Ramon F 1995: Magnetic resonance arthrography of the scapulohumeral joint in dogs, using gadopentetate dimeglumine. Am J Vet Res 56: 286-288

Winalski CS, Aliabadi P, Wright RJ, Shortkroff S, Sledge CB, Weissman BN 1993: Enhancement of joint fluid with intravenously administered gadopentetate dimeglumine: technique, rationale, and implications. Radiology 187: $179-185$ 
Plate IV

Zhalniarovich Y. et al.: Direct magnetic ... pp. 85-89
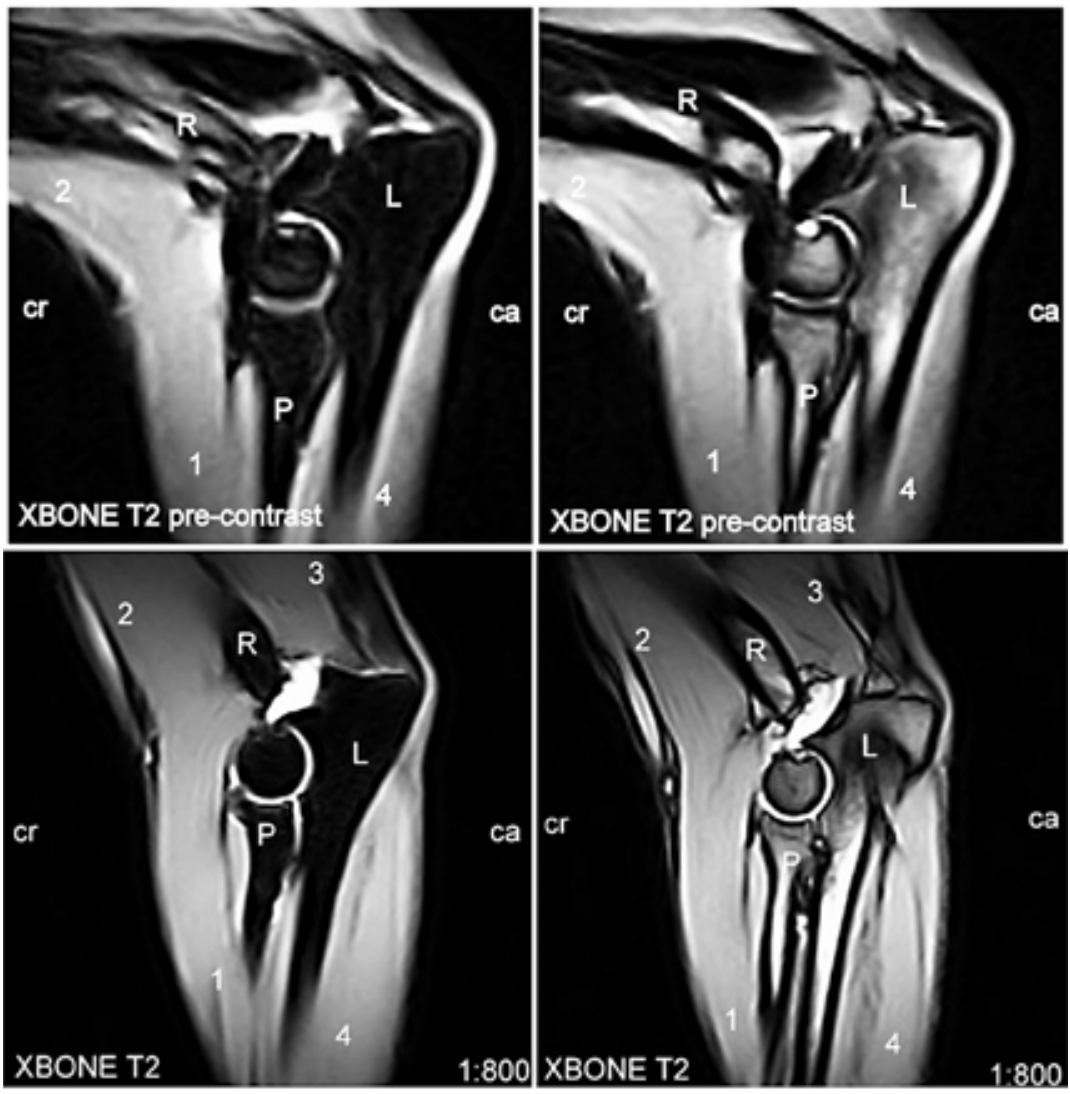

Fig. 1. XBONE T2 sequence in the sagittal plane pre- and after contrast administration. Gadolinium-containing contrast medium is diluted 1 to 800 . $\mathrm{R}$ - humerus; $\mathrm{L}$ - ulna; $\mathrm{P}$-radius; 1 - extensor carpi radialis muscle; 2 - biceps brachii muscle; 3 - triceps brachii muscle; 4 - flexor carpi radialis muscle. 




Fig. 2. XBONE T1 and 3D SST1 sequences in the transverse plane after the administration of the gadolinium contrast agent diluted 1:800. $\mathrm{R}$ - humerus; $\mathrm{L}$ - ulna; 1 - extensor digitorum communis muscle; 2 - extensor carpi radialis muscle; 3 - deltoid muscle; 4 - biceps brachii muscle; 5 - pronator teres muscle; 6 - flexor carpi radialis muscle.

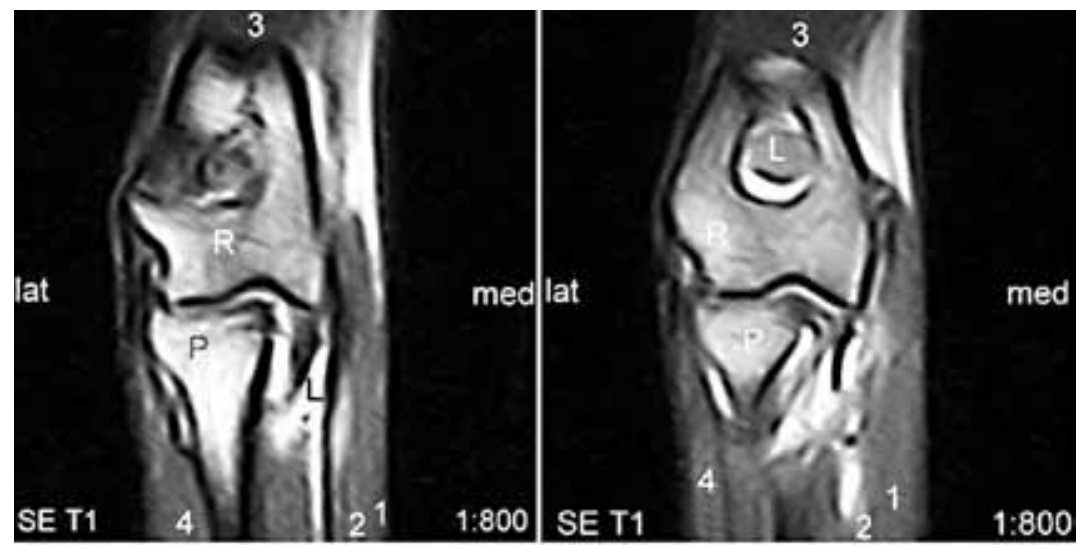

Fig. 3. Spin Echo T1 sequence in the dorsal plane after the administration of the gadolinium contrast agent diluted 1:800. $\mathrm{R}$ - humerus; $\mathrm{L}$ - ulna; $\mathrm{P}$ - radius; 1 - superficial digital flexor; 2 deep digital flexor; 3 - triceps brachii muscle; 4 - extensor digitorum lateralis muscle. 